Georgian Mathematical Journal

1(1994), No. 1, 31-45

\title{
ON TWO-POINT BOUNDARY VALUE PROBLEMS FOR SYSTEMS OF HIGHER-ORDER ORDINARY DIFFERENTIAL EQUATIONS WITH SINGULARITIES
}

\author{
I. KIGURADZE AND G. TSKHOVREBADZE
}

Abstract. The sufficient conditions of solvability and unique solvability of the two-point boundary value problems of Vallèe-Poussin and Cauchy-Niccoletti have been found for a system of ordinary differential equations of the form

$$
u^{(n)}=f\left(t, u, u^{\prime}, \ldots, u^{(n-1)}\right),
$$

where the vector function $f:] a, b\left[\times \mathbb{R}^{n l} \rightarrow \mathbb{R}^{l}\right.$ has nonintegrable singularities with respect to the first argument at the points $a$ and $b$.

\section{$\S 1$. Statement of the main Results}

In this paper for an $l$-dimensional system of differential equations

$$
u^{(n)}=f\left(t, u, u^{\prime}, \ldots, u^{(n-1)}\right)
$$

we consider the boundary value problem of Vallèe-Poussin

$$
\begin{gathered}
u(a+)=\cdots=u^{(m-1)}(a+)=0, \\
u(b-)=\cdots=u^{(n-m-1)}(b-)=0
\end{gathered}
$$

and that of Cauchy-Niccoletti

$$
\begin{gathered}
u(a+)=\cdots=u^{(m-1)}(a+)=0, \\
u^{(m)}(b-)=\cdots=u^{(n-1)}(b-)=0,
\end{gathered}
$$

where $l \geq 1, n \geq 2, m$ is an integer part of the number $\frac{n}{2},-\infty<a<b<$ $+\infty$, and the vector function $f:] a, b\left[\times \mathbb{R}^{n l} \rightarrow \mathbb{R}^{l}\right.$ satisfies the Caratheodory conditions on each compact contained in $] a, b\left[\times \mathbb{R}^{n l}\right.$. We are interested mainly in the singular case when $f$ is nonintegrable with respect to the first argument on $[a, b]$, having singularities at the ends of this interval. The above problems were investigated for $l=1$ in $[2-6]$.

1991 Mathematics Subject Classification. 34B15. 
The following notations will be used:

$$
\begin{gathered}
I_{n}(a, b)=\left\{\begin{array}{l}
] a, b[\text { for } n=2 m \\
] a, b] \text { for } n=2 m+1
\end{array} ;\right. \\
\mu_{n}=\left\{\begin{array}{r}
1 \text { for } n=2 m \\
\frac{n}{2} \text { for } n=2 m+1
\end{array} ;\right. \\
\lambda_{i m}(a, b ; t)=\frac{\min \left\{(t-a)^{2 m-i},(b-t)^{2 m-i}\right\}}{(m-1) !(m-i) ! \sqrt{(2 m-1)(2 m-2 i+1)}} \\
(i=1, \ldots, m) ;
\end{gathered}
$$

$\mathbb{R}$ is a set of real numbers, $\mathbb{R}_{+}=[0,+\infty[$;

$\xi=\left(\xi_{j}\right)_{j=1}^{l} \in \mathbb{R}^{l}$ and $A=\left(a_{k j}\right)_{k, j=1}^{l} \in \mathbb{R}^{l \times l}$ are respectively an $l$ dimensional column vector and an $l \times l$ matrix with real components $\xi_{j}(j=$ $1, \ldots, l)$ and $a_{k j}(k, j=1, \ldots, l)$,

$$
\begin{gathered}
|\xi|=\left(\left|\xi_{j}\right|\right)_{j=1}^{l}, \quad\|\xi\|=\sum_{j=1}^{l}\left|\xi_{j}\right|, \quad\|A\|=\sum_{k, j=1}^{l}\left|a_{k j}\right|, \\
S(\xi)=\left(\begin{array}{cccc}
\operatorname{sign} \xi_{1} & 0 & \ldots & 0 \\
0 & \operatorname{sign} \xi_{2} & \ldots & 0 \\
\ldots & \ldots & \ldots & \ldots \\
0 & 0 & \ldots & \operatorname{sign} \xi_{l}
\end{array}\right)
\end{gathered}
$$

$r(A)$ is the spectral radius of the matrix $A$;

$\mathbb{R}_{+}^{l}$ and $\mathbb{R}_{+}^{l \times l}$ are sets of $l$-dimensional vectors and $l \times l$ matrices with nonnegative components;

the inequalities $\xi \leq \bar{\xi}$ and $A \leq \bar{A}$, where $\xi$ and $\bar{\xi} \in \mathbb{R}^{l}$ and $A$ and $\bar{A} \in \mathbb{R}^{l \times l}$, imply respectively $\bar{\xi}-\xi \in \mathbb{R}_{+}^{l}$ and $\bar{A}-A \in \mathbb{R}_{+}^{l \times l}$;

$L_{\text {loc }}\left(I ; \mathbb{R}_{+}\right)$, where $I \subset \mathbb{R}$ is an interval, is a set of functions $x: I \rightarrow \mathbb{R}_{+}$ which are Lebesgue integrable on each segment contained in $I$;

$K_{\text {loc }}\left(I \times \mathbb{R}^{p} ; \mathbb{R}^{l}\right)$, where $p$ is a natural number, is a set of vector functions mapping $I \times \mathbb{R}^{p}$ into $\mathbb{R}^{l}$ and satisfying the Caratheodory conditions on each compact contained in $I \times \mathbb{R}^{p}$;

$\widetilde{C}_{l o c}^{p}\left(I ; \mathbb{R}^{l}\right)$ is a set of vector functions $u: I \rightarrow \mathbb{R}^{l}$ which are absolutely continuous together with all their derivatives up to order $p$ inclusive on each segment contained in $I$;

$\widetilde{C}^{n-1, m}\left(I ; \mathbb{R}^{l}\right)$ is a set of vector functions $u \in \widetilde{C}_{l o c}^{n-1}\left(I ; \mathbb{R}^{l}\right)$ satisfying the condition

$$
\int_{I}\left\|u^{(m)}(\tau)\right\|^{2} d \tau<+\infty .
$$

As mentioned above, throughout this paper it is assumed that

$$
f \in K_{l o c}(] a, b\left[\times \mathbb{R}^{n l} ; \mathbb{R}^{l}\right) .
$$


Theorem 1.1. Let the following inequalities be fulfilled on $] a, b\left[\times \mathbb{R}^{n l}\right.$ :

$$
(-1)^{n-m-1} S\left(x_{1}\right) f\left(t, x_{1}, \ldots, x_{n}\right) \geq-\sum_{i=1}^{m} H_{i}(t)\left|x_{i}\right|-h(t)
$$

and

$$
\left\|f\left(t, x_{1}, \ldots, x_{n}\right)\right\| \leq q\left(t, x_{1}, \ldots, x_{m}\right) \sum_{i=m+1}^{n}\left(1+\left\|x_{i}\right\|\right)^{\frac{2 n-2 m-1}{2 i-2 m-1}},
$$

where

$$
q \in K_{l o c}\left(I_{n}(a, b) \times \mathbb{R}^{m l} ; \mathbb{R}_{+}\right),
$$

and $\left.H_{i}:\right] a, b\left[\rightarrow \mathbb{R}_{+}^{l \times l}(i=1, \ldots, m)\right.$ and $\left.h:\right] a, b\left[\rightarrow \mathbb{R}_{+}^{l}\right.$ are respectively measurable matrix and vector functions satisfying the conditions

$$
\begin{gathered}
\int_{a}^{b}(\tau-a)^{n-m-\frac{1}{2}}(b-\tau)^{m-\frac{1}{2}}\|h(\tau)\| d \tau<+\infty \\
\int_{a}^{b}(\tau-a)^{n-i}(b-\tau)^{2 m-i}\left\|H_{i}(\tau)\right\| d \tau<+\infty \quad(i=1, \ldots, m), \\
r\left(\sum_{i=1}^{m} \int_{a}^{b}(\tau-a)^{n-2 m} \lambda_{i m}(a, b ; \tau) H_{i}(\tau) d \tau\right)<\mu_{n} .
\end{gathered}
$$

Then the problem $(1.1),(1.2)$ is solvable in the class $\widetilde{C}^{n-1, m}\left(I_{n}(a, b) ; \mathbb{R}^{l}\right)$.

Theorem 1.2. Let on $] a, b\left[\times \mathbb{R}^{n l}\right.$ the inequalities (1.4) and (1.5) be fulfilled, where $\left.\left.q \in K_{\text {loc }}(] a, b\right] \times \mathbb{R}^{m l} ; \mathbb{R}_{+}\right)$, and $\left.\left.H_{i}:\right] a, b\right] \rightarrow \mathbb{R}_{+}^{l \times l}(i=1, \ldots, m)$ and $h:[a, b] \rightarrow \mathbb{R}_{+}^{l}$ are respectively measurable matrix and vector functions satisfying the conditions

$$
\begin{gathered}
\int_{a}^{b}(\tau-a)^{n-m-\frac{1}{2}}\|h(\tau)\| d \tau<+\infty, \\
\int_{a}^{b}(\tau-a)^{n-i}\left\|H_{i}(\tau)\right\| d \tau<+\infty \quad(i=1, \ldots, m), \\
r\left(\sum_{i=1}^{m} \frac{1}{(m-1) !(m-i) ! \sqrt{(2 m-1)(2 m-2 i+1)}} \times\right. \\
\left.\times \int_{a}^{b}(\tau-a)^{n-i} H_{i}(\tau) d \tau\right)<\mu_{n} .
\end{gathered}
$$

Then the problem (1.1), (1.3) is solvable in the class $\left.\left.\widetilde{C}^{n-1, m}(] a, b\right] ; \mathbb{R}^{l}\right)$. 
For a differential system

$$
u^{(n)}=f\left(t, u, u^{\prime}, \ldots, u^{(m-1)}\right),
$$

not containing intermediate derivatives of order higher than $(m-1)$, Theorems 1.1 and 1.2 can be formulated as follows:

Theorem 1.1'. Let $f \in K_{l o c}\left(I_{n}(a, b) \times \mathbb{R}^{m l} ; \mathbb{R}^{l}\right)$ and on $] a, b\left[\times \mathbb{R}^{m l}\right.$

$$
(-1)^{n-m-1} S\left(x_{1}\right) f\left(t, x_{1}, \ldots, x_{m}\right) \geq-\sum_{i=1}^{m} H_{i}(t)\left|x_{i}\right|-h(t),
$$

where $\left.H_{i}:\right] a, b\left[\rightarrow \mathbb{R}_{+}^{l \times l}(i=1, \ldots, m)\right.$ and $\left.h:\right] a, b\left[\rightarrow \mathbb{R}_{+}^{l}\right.$ are measurable matrix and vector functions satisfying the conditions (1.7)-(1.9). Then the problem $\left(1.1^{\prime}\right),(1.2)$ is solvable in the class $\widetilde{C}^{n-1, m}\left(I_{n}(a, b) ; \mathbb{R}^{l}\right)$.

Theorem 1.2'. Let $\left.\left.f \in K_{l o c}(] a, b\right] \times \mathbb{R}^{m l} ; \mathbb{R}^{l}\right)$ and on $] a, b\left[\times \mathbb{R}^{m l}\right.$ the inequality $\left(1.4^{\prime}\right)$ be fulfilled, where $\left.\left.H_{i}:\right] a, b\right] \rightarrow \mathbb{R}_{+}^{l \times l}(i=1, \ldots, m)$ and $h:[a, b] \rightarrow \mathbb{R}_{+}^{l}$ are measurable matrix and vector functions satisfying the conditions (1.10)-(1.12). Then the problem (1.1'), (1.3)is solvable in the class $\left.\left.\widetilde{C}^{n-1, m}(] a, b\right] ; \mathbb{R}^{l}\right)$.

Theorem 1.3. Let

$$
\begin{aligned}
& f \in K_{l o c}\left(I_{n}(a, b) \times \mathbb{R}^{m l} ; \mathbb{R}^{l}\right) \\
& \int_{a}^{b}(\tau-a)^{n-m-\frac{1}{2}}(b-\tau)^{m-\frac{1}{2}}\|f(\tau, 0, \ldots, 0)\| d \tau<+\infty
\end{aligned}
$$

and on $] a, b\left[\times \mathbb{R}^{m l}\right.$

$$
\begin{gathered}
(-1)^{n-m-1} S\left(x_{1}-y_{1}\right)\left[f\left(t, x_{1}, \ldots, x_{m}\right)-f\left(t, y_{1}, \ldots, y_{m}\right)\right] \geq \\
\geq-\sum_{i=1}^{m} H_{i}(t)\left|x_{i}-y_{i}\right|
\end{gathered}
$$

where $\left.H_{i}:\right] a, b\left[\rightarrow \mathbb{R}_{+}^{l \times l}(i=1, \ldots, m)\right.$ are measurable matrix functions satisfying the conditions (1.8) and (1.9). Then the problem (1.1'), (1.2) is uniquely solvable in the class $\widetilde{C}^{n-1, m}\left(I_{n}(a, b) ; \mathbb{R}^{l}\right)$.

Theorem 1.4. Let

$$
\left.\left.f \in K_{l o c}(] a, b\right] \times \mathbb{R}^{m l} ; \mathbb{R}^{l}\right), \quad \int_{a}^{b}(\tau-a)^{n-m-\frac{1}{2}}\|f(\tau, 0, \ldots, 0)\| d \tau<+\infty
$$

and on $] a, b\left[\times \mathbb{R}^{m l}\right.$ the inequality (1.14) be fulfilled, where $\left.\left.H_{i}:\right] a, b\right] \rightarrow \mathbb{R}_{+}^{l \times l}$ $(i=1, \ldots, m)$ are measurable matrix functions satisfying the conditions (1.11) and (1.12). Then the problem $\left(1.1^{\prime}\right),(1.3)$ is uniquely solvable in the class $\left.\left.\widetilde{C}^{n-1, m}(] a, b\right] ; \mathbb{R}^{l}\right)$. 


\section{$\S$ 2. Auxiliary propositions}

Lemma 2.1. Let $I \subset \mathbb{R}$ be some interval, $k$ be a natural number, $\rho_{0} \in$ ] $0,+\infty[$ and

$$
\varphi \in L_{l o c}\left(I ; \mathbb{R}_{+}\right) .
$$

Then there exists a continuous function $\rho: I \rightarrow \mathbb{R}_{+}$such that for any vector function $v \in \widetilde{C}_{\text {loc }}^{k}\left(I ; \mathbb{R}^{l}\right)$ satisfying almost everywhere on I the differential inequality

$$
\left\|v^{(k+1)}(t)\right\| \leq \varphi(t)\left[1+\sum_{i=0}^{k}\left\|v^{(i)}(t)\right\|^{\frac{2 k+1}{2 i+1}}\right]
$$

and the condition

$$
\int_{I}\|v(\tau)\|^{2} d \tau \leq \rho_{0}^{2}
$$

the estimates

$$
\left\|v^{(i)}(t)\right\|<\rho(t) \quad \text { for } t \in I \quad(i=0, \ldots, k)
$$

hold.

Proof. In the case $I=[a, b]$ it is not difficult to verify by Lemma 2.2 from [6] that there exists a positive constant $\widetilde{\rho}$ such that the estimates $\left\|v^{(i)}(t)\right\|<\widetilde{\rho}$ for $a \leq t \leq b(i=0, \ldots, k)$ hold for any vector function $v \in \widetilde{C}_{l o c}^{k}\left(I ; \mathbb{R}^{l}\right)$ satisfying the conditions (2.2) and (2.3); in other words, we have (2.4), where $\rho(t) \equiv \widetilde{\rho}$.

Now consider the case $I=] a, b]$. Choose any decreasing sequence $a_{j} \in$ ]$a, b](j=0,1,2, \ldots)$ such that $a_{0}=b$ and $\lim _{j \rightarrow+\infty} a_{j}=a$. Then, by virtue of the above reasoning, for any natural number $j$ there exists a positive constant $\rho_{j}$ such that any vector function $v \in \widetilde{C}_{l o c}^{k}\left(I ; \mathbb{R}^{l}\right)$ satisfying the conditions (2.2) and (2.3) admits the estimates

$$
\left\|v^{(i)}(t)\right\|<\rho_{j} \text { for } a_{j} \leq t \leq b(i=0, \ldots, k) .
$$

Without loss of generality the sequence $\left(\rho_{j}\right)_{j=1}^{+\infty}$ can be assumed to be nondecreasing. Then (2.5) yields the estimates (2.4), where

$$
\rho(t)=\rho_{j}+\frac{t-a_{j-1}}{a_{j}-a_{j-1}}\left(\rho_{j+1}-\rho_{j}\right) \text { for } a_{j}<t \leq a_{j-1}(j=1,2, \ldots)
$$

with $\rho: I \rightarrow \mathbb{R}_{+}$being continuous and independent of $v$.

The cases $I=[a, b[$ and $I=] a, b[$ are considered similarly. 
Lemma 2.2. Let $\left.H_{i}:\right] a, b\left[\rightarrow \mathbb{R}_{+}^{l \times l}(i=1, \ldots, m)\right.$ and $\left.h:\right] a, b\left[\rightarrow \mathbb{R}_{+}^{l}\right.$ be measurable matrix and vector functions satisfying the conditions (1.7)-(1.9) and

$$
H=\sum_{i=1}^{m} \int_{a}^{b}(\tau-a)^{n-2 m} \lambda_{i m}(a, b ; \tau) H_{i}(\tau) d \tau .
$$

Then for any vector function $u \in \widetilde{C}^{n-1, m}(] a, b\left[; \mathbb{R}^{l}\right)$ satisfying a system of differential inequalities

$$
\begin{gathered}
(-1)^{n-m-1} S(u(t)) u^{(n)}(t) \geq-\sum_{i=1}^{m} H_{i}(t)\left|u^{(i-1)}(t)\right|-h(t) \\
\text { for } a<t<b
\end{gathered}
$$

and the boundary conditions (1.2) we have the estimates

$$
\int_{a}^{b}\left\|u^{(m)}(\tau)\right\|^{2} d \tau \leq \rho_{0}^{2}
$$

and

$$
\left\|u^{(i-1)}(t)\right\| \leq \rho_{0} \sigma_{i m}(a, b ; t) \quad \text { for } a<t<b(i=1, \ldots, m),
$$

where

$$
\begin{gathered}
\sigma_{i m}(a, b ; t)=\frac{\min \left\{(t-a)^{m-i+\frac{1}{2}},(b-t)^{m-i+\frac{1}{2}}\right\}}{(m-i) ! \sqrt{2 m-2 i+1}}, \\
\rho_{0}=\sqrt{l}\left\|\left(\mu_{n} E-H\right)^{-1}\right\| \times \\
\times \int_{a}^{b}(\tau-a)^{n-2 m} \sigma_{1 m}(a, b ; \tau)\|h(\tau)\| d \tau
\end{gathered}
$$

and $E$ is the unit $l \times l$ matrix.

To prove this lemma we need

Lemma 2.3. Let

$$
w(t)=\sum_{i=1}^{n-m} \sum_{k=i}^{n-m} c_{i k}(t) v^{(n-k)}(t) v^{(i-1)}(t),
$$

where

$$
\begin{gathered}
v \in \widetilde{C}^{n-1, m}(] a, b[; \mathbb{R}), \quad v^{(i-1)}(a+)=0 \quad(i=1, \ldots, m), \\
v^{(j-1)}(b-)=0 \quad(j=1, \ldots, n-m)
\end{gathered}
$$


and each $c_{i k}:[a, b] \rightarrow \mathbb{R}$ is a $(n-k-i+1)$-times continuously differentiable function; in that case there exists a positive constant $c_{0}$ such that

$$
\begin{gathered}
\left|c_{i i}(t)\right| \leq c_{0}(t-a)^{n-2 m} \text { for } a \leq t \leq b \\
(i=1, \ldots, n-m) .
\end{gathered}
$$

Then

$$
\lim _{t \rightarrow a+} \inf |w(t)|=0, \quad \lim _{t \rightarrow b-} \inf |w(t)|=0 .
$$

Proof. In the first place it will be shown that

$$
\lim _{t \rightarrow a+} \inf |w(t)|=0 .
$$

Let the opposite be true. Then without loss of generality one may assume that the inequality $w(t) \geq \delta$ for $a<t \leq a+2 \varepsilon_{0}$ is fulfilled for some $\delta \in] 0,+\infty\left[\right.$ and $\left.\varepsilon_{0} \in\right] 0, \frac{b-a}{4}[\cap] 0,1[$.

Therefore

$$
\begin{gathered}
\sum_{i=1}^{n-m} \sum_{k=i}^{n-m} q_{i k}(t ; \varepsilon) v^{(n-k)}(t) v^{(i-1)}(t) \geq \delta(t-a-\varepsilon)^{n}(a+2 \varepsilon-t)^{n} \\
\quad \text { for } \quad a+\varepsilon \leq t \leq a+2 \varepsilon, \quad 0<\varepsilon \leq \varepsilon_{0}
\end{gathered}
$$

where $q_{i k}(t ; \varepsilon)=(t-a-\varepsilon)^{n}(a+2 \varepsilon-t)^{n} c_{i k}(t)$. After integrating the latter inequality from $a+\varepsilon$ to $a+2 \varepsilon$ according to Lemma 4.1 from [7], we obtain

$$
\begin{gathered}
\sum_{i=1}^{n-m} \sum_{k=i}^{n-m} \sum_{j=0}^{m_{i k}} \nu_{i k j} \int_{a+\varepsilon}^{a+2 \varepsilon} q_{i k}^{(n-k-i-2 j+1)}(\tau ; \varepsilon)\left[v^{(i+j-1)}(\tau)\right]^{2} d \tau \geq \\
\geq \delta \int_{a+\varepsilon}^{a+2 \varepsilon}(\tau-a-\varepsilon)^{n}(a+2 \varepsilon-\tau)^{n} d \tau,
\end{gathered}
$$

where $m_{i k}$ is the integer part of the number $\frac{1}{2}(n-k-i+1)$ and $\nu_{i k j}$ $\left(i=1, \ldots, n-m ; k=i, \ldots, n-m ; j=0, \ldots, m_{i k}\right)$ are positive constants independent of $a, \varepsilon$ and $v$.

If $k \in\{i+1, \ldots, n-m\}$, then we have $i+j-1 \leq m-1,2 n-(n-k-$ $i-2 j+1) \geq 2 i+2 j+n$ for any $j \in\left\{0, \ldots, m_{i k}\right\}$.

Therefore, taking into account (2.11) and (2.14), we find

$$
\begin{aligned}
{\left[v^{(i+j-1)}(t)\right]^{2} } & =\left[\frac{1}{(m-i-j) !} \int_{a}^{t}(t-\tau)^{m-i-j} v^{(m)}(\tau) d \tau\right]^{2} \leq \\
\leq & \alpha(\varepsilon) \varepsilon^{2 m-2 i-2 j+1} \text { for } a<t \leq a+2 \varepsilon
\end{aligned}
$$


and $\left|q_{i k}^{(n-k-i-2 j+1)}(t ; \varepsilon)\right| \leq \alpha_{1} \varepsilon^{2 i+2 j+n}$ for $a \leq t \leq a+2 \varepsilon$, where

$$
\alpha(\varepsilon)=2^{2 m-1} \int_{a}^{a+2 \varepsilon}\left[v^{(m)}(\tau)\right]^{2} d \tau \rightarrow 0 \text { for } \varepsilon \rightarrow 0
$$

and $\alpha_{1}$ is a positive constant independent of $\varepsilon$. Therefore

$$
\begin{gathered}
\left|\int_{a+\varepsilon}^{a+2 \varepsilon} q_{i k}^{(n-k-i-2 j+1)}(\tau ; \varepsilon)\left[v^{(i+j-1)}(\tau)\right]^{2} d \tau\right| \leq \alpha_{1} \alpha(\varepsilon) \varepsilon^{2 m+2+n} \leq \\
\leq \alpha_{1} \alpha(\varepsilon) \varepsilon^{2 n+1} .
\end{gathered}
$$

Consider now the case $k=i$. By virtue of (2.12) and (2.14) we have

$$
\begin{gathered}
\left|q_{i k}^{(n-k-i-2 j+1)}(t ; \varepsilon)\right|=\left|q_{i i}^{(n-2 i-2 j+1)}(t ; \varepsilon)\right| \leq \alpha_{2} \varepsilon^{2 n-2 m+2 i+2 j-1} \\
\text { for } a \leq t \leq a+2 \varepsilon
\end{gathered}
$$

where $\alpha_{2}$ is a positive constant independent of $\varepsilon$. Therefore if $i+j-1=m$, then

$$
\begin{aligned}
& \left|\int_{a+\varepsilon}^{a+2 \varepsilon} q_{i i}^{(n-2 i-2 j+1)}(\tau ; \varepsilon)\left[v^{(i+j-1)}(\tau)\right]^{2} d \tau\right|= \\
= & \left|\int_{a+\varepsilon}^{a+2 \varepsilon} q_{i i}(\tau ; \varepsilon)\left[v^{(m)}(\tau)\right]^{2} d \tau\right| \leq \alpha_{2} \alpha(\varepsilon) \varepsilon^{2 n+1},
\end{aligned}
$$

if however $i+j-1<m$, then, taking into account (2.16), we obtain

$$
\left|\int_{a+\varepsilon}^{a+2 \varepsilon} q_{i i}^{(n-2 i-2 j+1)}(\tau ; \varepsilon)\left[v^{(i+j-1)}(\tau)\right]^{2} d \tau\right| \leq \alpha_{2} \alpha(\varepsilon) \varepsilon^{2 n+1} .
$$

Thus

$$
\begin{gathered}
\left|\int_{a+\varepsilon}^{a+2 \varepsilon} q_{i k}^{(n-k-i-2 j+1)}(\tau ; \varepsilon)\left[v^{(i+j-1)}(\tau)\right]^{2} d \tau\right| \leq \alpha_{0} \alpha(\varepsilon) \varepsilon^{2 n+1}, \\
\quad\left(i=1, \ldots, n-m ; k=i, \ldots, n-m, \quad j=0, \ldots, m_{i k}\right)
\end{gathered}
$$

where $\alpha_{0}=\max \left\{\alpha_{1}, \alpha_{2}\right\}$.

On the other hand,

$$
\begin{aligned}
\int_{a+\varepsilon}^{a+2 \varepsilon}(\tau-a-\varepsilon)^{n}(a+2 \varepsilon-\tau)^{n} d \tau & \geq \frac{\varepsilon^{n}}{2^{n}} \int_{a+\varepsilon}^{a+\frac{3 \varepsilon}{2}}(\tau-a-\varepsilon)^{n} d \tau= \\
& =\frac{1}{2^{2 n+1}(n+1)} \varepsilon^{2 n+1} .
\end{aligned}
$$

Due to (2.18) and the latter inequality we find from (2.15) that $\alpha(\varepsilon) \geq \delta_{0}$ for $0<\varepsilon \leq \varepsilon_{0}$, where $\delta_{0}$ is a positive constant independent of $\varepsilon$. But the latter inequality contradicts the condition (2.17). This contradiction proves that (2.13) holds. 
The equality $\lim _{t \rightarrow b-} \inf |w(t)|=0$ is proved similarly, the only difference being that for $n=2 m+1$ instead of $(2.12)$ the condition $v^{(m)}(b-)=0$ is used.

Proof of Lemma 2.2. For each component $u_{j}(j=1, \ldots, l)$ of the solution $u$ of the problem (2.7), (1.2) we have

$$
\begin{gathered}
\left|u_{j}^{(i-1)}(t)\right|=\left|\frac{1}{(m-i) !} \int_{a}^{t}(t-\tau)^{m-i} u_{j}^{(m)}(\tau) d \tau\right| \leq \\
\leq \frac{1}{(m-i) ! \sqrt{2 m-2 i+1}}(t-a)^{m-i+\frac{1}{2}}\left(\int_{a}^{b}\left[u_{j}^{(m)}(\tau)\right]^{2} d \tau\right)^{\frac{1}{2}} \\
\text { for } a<t<b \quad(i=1, \ldots, m)
\end{gathered}
$$

and

$$
\begin{gathered}
\left|u_{j}^{(i-1)}(t)\right|=\left|\frac{1}{(m-i) !} \int_{t}^{b}(\tau-t)^{m-i} u_{j}^{(m)}(\tau) d \tau\right| \leq \\
\leq \frac{1}{(m-i) ! \sqrt{2 m-2 i+1}}(b-t)^{m-i+\frac{1}{2}}\left(\int_{a}^{b}\left[u_{j}^{(m)}(\tau)\right]^{2} d \tau\right)^{\frac{1}{2}} \\
\text { for } a<t<b \quad(i=1, \ldots, m) .
\end{gathered}
$$

Therefore

$$
\left|u_{j}^{(i-1)}(t)\right| \leq \sigma_{i m}(a, b ; t) \rho_{j} \text { for } a<t<b(i=1, \ldots, m),
$$

where

$$
\rho_{j}=\left(\int_{a}^{b}\left[u_{j}^{(m)}(\tau)\right]^{2} d \tau\right)^{\frac{1}{2}} .
$$

Let $H_{i}(t)=\left(h_{i j k}(t)\right)_{j, k=1}^{l}(i=1, \ldots, m), \quad h(t)=\left(h_{j}(t)\right)_{j=1}^{l}$. Rewrite (2.7) in terms of components as

$$
\begin{gathered}
(-1)^{n-m-1} u_{j}^{(n)}(t) \operatorname{sign} u_{j}(t) \geq \\
\geq-\sum_{i=1}^{m} \sum_{k=1}^{l} h_{i j k}(t)\left|u_{k}^{(i-1)}(t)\right|-h_{j}(t) \quad(j=1, \ldots, l) .
\end{gathered}
$$


After multiplying both sides of $\left(2.7^{\prime}\right)$ by $(t-a)^{n-2 m}\left|u_{j}(t)\right|$ and integrating from $s$ to $t$, we obtain

$$
\begin{gathered}
(-1)^{n-m} \int_{s}^{t}(\tau-a)^{n-2 m} u_{j}^{(n)}(\tau) u_{j}(\tau) d \tau \leq \\
\leq \sum_{i=1}^{m} \sum_{k=1}^{l} \int_{s}^{t}(\tau-a)^{n-2 m} h_{i j k}(\tau)\left|u_{k}^{(i-1)}(\tau)\right|\left|u_{j}(\tau)\right| d \tau+ \\
+\int_{s}^{t}(\tau-a)^{n-2 m} h_{j}(\tau)\left|u_{j}(\tau)\right| d \tau \text { for } a<s \leq t<b .
\end{gathered}
$$

By virtue of (2.19)

$$
\begin{gathered}
\sum_{k=1}^{l} \int_{s}^{t}(\tau-a)^{n-2 m} h_{i j k}(\tau)\left|u_{k}^{(i-1)}(\tau)\right|\left|u_{j}(\tau)\right| d \tau \leq \\
\leq \rho_{j} \sum_{k=1}^{l} \rho_{k} \int_{s}^{t}(\tau-a)^{n-2 m} \sigma_{1 m}(a, b ; \tau) \sigma_{i m}(a, b ; \tau) h_{i j k}(\tau) d \tau= \\
=\rho_{j} \sum_{k=1}^{l} \rho_{k} \int_{s}^{t}(\tau-a)^{n-2 m} \lambda_{i m}(a, b ; \tau) h_{i j k}(\tau) d \tau \\
\int_{s}^{t}(\tau-a)^{n-2 m} h_{i}(\tau)\left|u_{j}(\tau)\right| d \tau \leq \\
\leq \rho_{j} \int_{s}^{t}(\tau-a)^{n-2 m} \sigma_{1 m}(a, b ; \tau) h_{j}(\tau) d \tau .
\end{gathered}
$$

On the other hand, by Lemma 4.1 from [7]

$$
\begin{gathered}
\int_{s}^{t}(\tau-a)^{n-2 m} u_{j}^{(n)}(\tau) u_{j}(\tau) d \tau= \\
=w_{j}(t)-w_{j}(s)+(-1)^{n-m} \mu_{n} \int_{s}^{t}\left[u_{j}^{(m)}(\tau)\right]^{2} d \tau,
\end{gathered}
$$

where

$$
w_{j}(t)= \begin{cases}\sum_{p=1}^{n-m}(-1)^{p-1} u_{j}^{(n-p)}(t) u_{j}^{(p-1)}(t) & \text { for } n=2 m, \\ \sum_{p=1}^{n-m-1}(-1)^{p-1}\left[(t-a) u_{j}^{(n-p)}(t)-\right. & \\ \left.-p u_{j}^{(n-p-1)}(t)\right] u_{j}^{(p-1)}(t)+(-1)^{m} \frac{t-a}{2}\left[u_{j}^{(m)}(t)\right]^{2} & \text { for } n=2 m+1 .\end{cases}
$$


As one may readily verify, the functions $w_{j}(j=1, \ldots, l)$ satisfy the conditions of Lemma 2.3 and therefore

$$
\lim _{s \rightarrow a+} \inf \left|w_{j}(s)\right|=0, \quad \lim _{t \rightarrow b-} \inf \left|w_{j}(t)\right|=0 \quad(j=1, \ldots, l) .
$$

Taking into account the latter equalities and conditions (1.7) and (1.8) from (2.20)-(2.23) we obtain

$$
\begin{gathered}
\mu_{n} \rho_{j}^{2} \leq \rho_{j} \sum_{i=1}^{m} \sum_{k=1}^{l} \rho_{k} \int_{a}^{b}(\tau-a)^{n-2 m} \lambda_{i m}(a, b ; \tau) h_{i j k}(\tau) d \tau+ \\
\quad+\rho_{j} \int_{a}^{b}(\tau-a)^{n-2 m} \sigma_{1 m}(a, b ; \tau) h_{j}(\tau) d \tau \quad(j=1, \ldots, l) .
\end{gathered}
$$

Hence by virtue of (2.6) we have

$$
\mu_{n} \rho \leq H \rho+\int_{a}^{b}(\tau-a)^{n-2 m} \sigma_{1 m}(a, b ; \tau) h(\tau) d \tau,
$$

where $\rho=\left(\rho_{j}\right)_{j=1}^{l}$. In view of (1.9) and the notation (2.10) from the latter inequality we find

$$
\rho \leq\left(\mu_{n} E-H\right)^{-1} \int_{a}^{b}(\tau-a)^{n-2 m} \sigma_{1 m}(a, b ; \tau) h(\tau) d \tau
$$

and

$$
\|\rho\| \leq\left\|\left(\mu_{n} E-H\right)^{-1}\right\| \int_{a}^{b}(\tau-a)^{n-2 m} \sigma_{1 m}(a, b ; \tau)\|h(\tau)\| d \tau=l^{-\frac{1}{2}} \rho_{0} .
$$

Hence

$$
\int_{a}^{b}\left\|u^{(m)}(\tau)\right\|^{2} d \tau \leq l\|\rho\|^{2} \leq \rho_{0}^{2} .
$$

On the other hand, in view of (2.19)

$$
\begin{gathered}
\left\|u^{(i-1)}(t)\right\| \leq \sigma_{i m}(a, b ; t)\|\rho\| \leq \rho_{0} \sigma_{i m}(a, b ; t) \quad \text { for } \quad a<t<b \\
(i=1, \ldots, m) .
\end{gathered}
$$

Therefore, the estimates (2.8) and (2.9) hold.

In a similar manner we prove

Lemma 2.4. Let $\left.\left.H_{i}:\right] a, b\right] \rightarrow \mathbb{R}_{+}^{l \times l}(i=1, \ldots, m)$, and let $\left.\left.h:\right] a, b\right] \rightarrow \mathbb{R}_{+}^{l}$ be measurable matrix and vector functions satisfying the conditions (1.10)(1.12). Then for any solution $\left.\left.u \in \widetilde{C}^{n-1, m}(] a, b\right] ; \mathbb{R}^{l}\right)$ of the problem (2.7), (1.3) we have the estimates

$$
\int_{a}^{b}\left\|u^{(m)}(\tau)\right\|^{2} d \tau \leq \rho_{0}^{2}
$$




$$
\begin{aligned}
& \text { and }\left\|u^{(i-1)}(t)\right\| \leq \rho_{0}(t-a)^{m-i+\frac{1}{2}} \text { for } a<t<b(i=1, \ldots, m) \text {, where } \\
& \rho_{0}=\frac{\sqrt{l}}{(m-1) ! \sqrt{2 m-1}}\left\|\left(\mu_{n} E-H\right)^{-1}\right\| \int_{a}^{b}(\tau-a)^{n-m-\frac{1}{2}}\|h(\tau)\| d \tau, \\
& H=\sum_{i=1}^{m} \frac{1}{(m-1) !(m-i) ! \sqrt{(2 m-1)(2 m-2 i+1)}} \int_{a}^{b}(\tau-a)^{n-i} H_{i}(\tau) d \tau,
\end{aligned}
$$

and $E$ is the unit $l \times l$ matrix.

\section{$\S 3$. Proof of the MAIN RESUlts}

Proof of Theorem 1.1. Let $\rho_{0}$ and $\sigma_{i m}(a, b ; t)(i=1, \ldots, m)$ be respectively the number and functions from Lemma 2.2 and

$$
\begin{gathered}
\varphi(t)=4^{n} \sup \left\{q\left(t, x_{1}, \ldots, x_{m}\right):\left\|x_{i}\right\| \leq \rho_{0} \sigma_{i m}(a, b ; t)\right. \\
(i=1, \ldots, m)\} .
\end{gathered}
$$

Then due to (1.6), (2.1) holds with $I=I_{n}(a, b)$.

For $k=n-m-1, \rho_{0}$, and $\varphi$, by virtue of Lemma 2.1 there exists a continuous function $\rho: I_{n}(a, b) \rightarrow \mathbb{R}_{+}$such that estimates (2.4) are valid for any vector function $v \in \widetilde{C}_{l o c}^{k}\left(I_{n}(a, b) ; \mathbb{R}^{l}\right)$ satisfying the conditions $(2.2)$ and (2.3).

Let

$$
\rho_{i}(t)= \begin{cases}\rho_{0} \sigma_{i m}(a, b ; t) & \text { for } \quad i \in\{1, \ldots, m\} \\ \rho(t) & \text { for } \quad i \in\{m+1, \ldots, n\}\end{cases}
$$

and $f^{*}(t)=\sup \left\{\left\|f\left(t, x_{1}, \ldots, x_{n}\right)\right\|:\left\|x_{i}\right\| \leq \rho_{i}(t)(i=1, \ldots, n)\right\}$. For any $i \in\{1, \ldots, n\}$ and $\xi=\left(\xi_{p}\right)_{p=1}^{l}$ we set

$$
\begin{aligned}
& \chi_{i p}(t, \xi)=\left\{\begin{array}{lll}
\xi_{p} & \text { for } & \left|\xi_{p}\right| \leq \rho_{i}(t) \\
\rho_{i}(t) \operatorname{sign} \xi_{p} & \text { for } & \left|\xi_{p}\right|>\rho_{i}(t)
\end{array},\right. \\
& \chi_{i}(t, \xi)=\left(\chi_{i p}(t, \xi)\right)_{p=1}^{l} .
\end{aligned}
$$

Let $j$ be an arbitrary natural number,

$$
\begin{gathered}
I_{n j}(a, b)=\left\{\begin{array}{ll}
{\left[a+\frac{b-a}{3 j}, b-\frac{b-a}{3 j}\right]} & \text { for } n=2 m \\
{\left[a+\frac{b-a}{3 j}, b\right]} & \text { for } n=2 m+1
\end{array},\right. \\
f_{j}\left(t, x_{1}, \ldots, x_{n}\right)= \\
=\left\{\begin{array}{lll}
f\left(t, \chi_{1}\left(t, x_{1}\right), \ldots, \chi_{n}\left(t, x_{n}\right)\right) & \text { for } t \in I_{n j}(a, b) \\
0 & \text { for } t \in[a, b] \backslash I_{n j}(a, b)
\end{array}\right.
\end{gathered}
$$




$$
f_{j}^{*}(t)=\left\{\begin{array}{ll}
f^{*}(t) & \text { for } \quad t \in I_{n j}(a, b) \\
0 & \text { for } \quad t \in[a, b] \backslash I_{n j}(a, b)
\end{array} .\right.
$$

Clearly, that $f_{j}^{*}:[a, b] \rightarrow \mathbb{R}_{+}$is the Lebesgue integrable function and on the $[a, b] \times \mathbb{R}^{n l}$ the inequality $\left\|f_{j}\left(t, x_{1}, \ldots, x_{n}\right)\right\| \leq f_{j}^{*}(t)$ holds. On the other hand the homogeneous differential system $u^{(n)}=0$ by boundary conditions (1.2) has only the trivial solution. Therefore by virtue of the Conti theorem [1] ${ }^{1}$ the differential system $u^{(n)}=f_{j}\left(t, u, \ldots, u^{(n-1)}\right)$ has a solution $u_{j} \in \widetilde{C}_{l o c}^{n-1}\left([a, b] ; \mathbb{R}^{l}\right)$ satisfying the boundary conditions (1.2). It is obvious that $u_{j} \in \widetilde{C}^{n-1, m}\left([a, b] ; \mathbb{R}^{l}\right)$. Simultaneously, from (1.4), (3.3), and (3.4) it follows that $u_{j}$ is the solution of the system of the differential inequalities (2.7). Therefore by virtue of Lemma 2.2

$$
\int_{a}^{b}\left\|u_{j}^{(m)}(\tau)\right\|^{2} d \tau \leq \rho_{0}^{2}
$$

and

$$
\left\|u_{j}^{(i-1)}(t)\right\| \leq \rho_{0} \sigma_{i m}(a, b ; t) \text { for } a<t<b \quad(i=1, \ldots, m) .
$$

From conditions (1.5) and (3.1)-(3.6) it is clear that the vector function $v_{j}(t)=u_{j}^{(m)}(t)$ satifies the inequalities (2.2) and (2.3). Hence by Lemma 2.1 $\left\|u_{j}^{(i-1)}(t)\right\|<\rho(t)$ for $t \in I_{n}(a, b)(i=m+1, \ldots, n)$. Therefore

$$
\left\|u_{j}^{(i-1)}(t)\right\|<\rho_{i}(t) \text { for } a<t<b(i=1, \ldots, n)
$$

and

$$
\left\|u_{j}^{(n)}(t)\right\| \leq f^{*}(t) \text { for } a<t<b .
$$

Moreover, in view of (3.3),(3.4) and (3.7) it is clear that

$$
u_{j}^{(n)}(t)=f\left(t, u_{j}(t), \ldots, u_{j}^{(n-1)}(t)\right) \text { for } t \in I_{n j}(a, b) .
$$

Since $f^{*} \in L_{l o c}\left(I_{n}(a, b) ; \mathbb{R}_{+}\right)$, the estimates (3.7) and (3.8) imply that the sequences $\left(u_{j}^{(i-1)}\right)_{j=1}^{+\infty}(i=1, \ldots, n)$ are uniformly bounded and equicontinuous on each segment contained in $I_{n}(a, b)$. Therefore, by virtue of the Arcela-Ascoli lemma these sequences can be regarded without loss of generality as uniformly converging on each segment from $I_{n}(a, b)$.

If we set $\lim _{j \rightarrow+\infty} u_{j}(t)=u(t)$ for $t \in I_{n}(a, b)$, then

$$
\lim _{j \rightarrow+\infty} u_{j}^{(i-1)}(t)=u^{(i-1)}(t) \text { for } t \in I_{n}(a, b)(i=1, \ldots, n)
$$

\footnotetext{
${ }^{1}$ See also [8], Corollary 2.1.
} 
uniformly on each segment contained in $I_{n}(a, b)$. Therefore from (3.5) and (3.6) we obtain

$$
\begin{gathered}
\int_{a}^{b}\left\|u^{(m)}(\tau)\right\|^{2} d \tau \leq \rho_{0}^{2}, \\
\left\|u^{(i-1)}(t)\right\| \leq \rho_{0} \sigma_{i m}(a, b ; t) \text { for } t \in I_{n}(a, b) \\
(i=1, \ldots, m) .
\end{gathered}
$$

In view of (3.9) for arbitrary fixed $s$ and $t \in I_{n}(a, b)$ there exists a natural number $j_{0}$ such that

$$
\begin{aligned}
u_{j}^{(n-1)}(t)-u_{j}^{(n-1)}(s) & =\int_{s}^{t} f\left(\tau, u_{j}(\tau), \ldots, u_{j}^{(n-1)}(\tau)\right) d \tau \\
(j & \left.=j_{0}, j_{0}+1, \ldots\right)
\end{aligned}
$$

and $s, t \in I_{n j}(a, b)$ for $j \geq j_{0}$. Passing to the limit in the latter equality by $j \rightarrow+\infty$, we obtain

$$
u^{(n-1)}(t)-u^{(n-1)}(s)=\int_{s}^{t} f\left(\tau, u(\tau), \ldots, u^{(n-1)}(\tau)\right) d \tau .
$$

Therefore $u$ is the solution of the system (1.1). Simultaneously, (3.10)-(3.12) imply that $u \in \widetilde{C}^{n-1, m}\left(I_{n}(a, b) ; \mathbb{R}^{l}\right)$ and satisfies the boundary conditions (1.2).

Theorem 1.1' immediately follows from Theorem 1.1, since in the case where $f\left(t, x_{1}, \ldots, x_{n}\right) \equiv f\left(t, x_{1}, \ldots, x_{m}\right)$ and $f \in K_{l o c}\left(I_{n}(a, b) \times \mathbb{R}^{m l} ; \mathbb{R}^{l}\right)$, inequality (1.5) is fulfilled automatically and the function $q\left(t, x_{1}, \ldots, x_{m}\right) \equiv$ $\left\|f\left(t, x_{1}, \ldots, x_{m}\right)\right\|$ satisfies the condition (1.6).

Proof of Theorem 1.3. (1.13) and (1.14) yield the conditions $\left(1.4^{\prime}\right)$ and $(1.7)$, where $h(t)=|f(t, 0, \ldots, 0)|$. Therefore by virtue of Theorem $1.1^{\prime}$ the problem $\left(1.1^{\prime}\right),(1.2)$ is solvable in the class $\widetilde{C}^{n-1, m}\left(I_{n}(a, b) ; \mathbb{R}^{l}\right)$.

To complete the proof of the theorem it remains for us to verify that the problem under consideration has at most one solution in the class $\widetilde{C}^{n-1, m}\left(I_{n}(a, b) ; \mathbb{R}^{l}\right)$.

Let $u, \bar{u} \in \widetilde{C}^{n-1, m}\left(I_{n}(a, b) ; \mathbb{R}^{l}\right)$ be two arbitrary solutions of the problem $\left(1.1^{\prime}\right),(1.2)$. We set $v(t)=u(t)-\bar{u}(t)$ for $t \in I_{n}(a, b)$.

It is clear that $v \in \widetilde{C}^{n-1, m}\left(I_{n}(a, b) ; \mathbb{R}^{l}\right)$ and $v(a+)=\cdots=v^{(m-1)}(a+)=$ $0, v(b-)=\cdots=v^{(n-m-1)}(b-)=0$.

On the other hand, by the condition (1.14) from the equality

$$
v^{(n)}(t)=f\left(t, u(t), \ldots, u^{(n-1)}(t)\right)-f\left(t, \bar{u}(t), \ldots, \bar{u}^{(n-1)}(t)\right)
$$


we have

$$
(-1)^{n-m-1} S(v(t)) v^{(n)}(t) \geq-\sum_{i=1}^{m} H_{i}(t)\left|v^{(i-1)}(t)\right| .
$$

Therefore due to Lemma $2.2 v(t) \equiv 0$, i.e., $u(t) \equiv \bar{u}(t)$.

Theorems 1.2 and $1.2^{\prime}$ are proved similarly to Theorems 1.1 and $1.1^{\prime}$, while Theorem 1.4 is proved similarly to Theorem 1.3 with the only difference being that Lemma 2.4 is used instead of Lemma 2.2.

\section{REFERENCES}

1. R. Conti, Equazioni differenziali oridinarie quasilineari con condizioni lineari. Ann. Mat. Pura Appl. 57(1962), 49-61.

2. I. T. Kiguradze, Some singular boundary value problems for ordinary differential equations. (Russian) Tbilisi University Press, Tbilisi, 1975.

3. — - On some singular boundary value problems for ordinary differential equations. Equadiff, 5. Proc. Czech. Conf. Diff. Equations and Applications, 174-178, Teubner Verlag, Leipzig, 1982.

4. I. T. Kiguradze, On solvability of boundary value problems of de la Vallèe Poussin. (Russian) Differentsial'nie Uravneniya 21(1985), No. 3, 391-398.

5. - On boundary value problems for high order ordinary differential equations with singularities. (Russian) Uspekhi Mat. Nauk 41(1986), No. 4, 166-167.

6. —- On two-point singular boundary value problems for nonlinear ordinary differential equations. (Russian) Proceedings of All-Union symposium on current problems of mathematical physics, Vol. 1 (Russian), 276-279, Tbilisi University Press, Tbilisi, 1987.

7. I. T. Kiguradze and T. A. Chanturia, Asymptotic properties of solutions of nonautonomous ordinary differential equations. (Russian) Nauka, Moscow, 1990.

8. I. T. Kiguradze, Boundary value problems for systems of ordinary differential equations. (Russian) Current problems in mathematics. Newest results, vol. 30 (Russian), 3-103, Itogi Nauki i Tekhniki, Akad. Nauk SSSR, Vses. Inst. Nauchn. i Tekhn. Inform., Moscow, 1987.

(Received 23.09.1992)

Authors' address:

A.Razmadze Mathematical Institute

Georgian Academy of Sciences

1 Rukhadze St., Tbilisi 380093

Republic of Georgia 